\title{
La trayectoria de la asistencia social en Brasil: atención a la familia y el lugar de la psicología*
}

\author{
The journey os social assistance in Brazil: \\ Attention to the family and the place of psychology
}

\author{
Lílian Rodrigues da Cruz** \\ Maria de Lourdes \\ Duque-Estrada Scarparo \\ Neuza Maria de Fátima Guareschi \\ Universidad de Santa Cruz do Sul
}

Recibido: Marzo 15 de 2007

Revisado: Abril 20 de 2007

Aceptado: J unio 24 de 2007

\section{Resumen}

El presente artículo tiene por objeto recorrer la trayectoria de las políticas sociales públicas en Brasil con foco en la constitución de la política de asistencia social. Considerando que desde la Constitución Federal de 1988 hasta el actual Sistema Único de Asistencia Social la familia tiene prioridad en Ley, centramos nuestra discusión en los programas sociales de atención a la familia, trayendo problematizaciones para las prácticas de la psicología.

Palabras clave: Políticas sociales públicas, asistencia social, familia, psicología.

\section{Abstract}

The present article aims to travel the path of the public social politics in Brazil, focusing the constitution of the social attendance politics. Considering that since the Federal Constitution of 1988 to the current

* $\quad$ Artículo traducido por María Piedad Rangel Meneses.

* Correspondencia: Lílian Rodriguez da Cruz, departamento de Psicología y Maestría en Letras, Universidad de Santa Cruz do Sul (UNISC). Dirección postal: Av. Salvador França, 1070 apto. 320, Bairro Jardim Botânico, Porto Alegre, RS, Brasil, CEP: $96690-000$. Correo electrónico: Iiliancruz2@terra.com.br. Maria de Lourdes Duque-Estrada Scarparo, maestría en Psicología Social e Institucional (UFRGS), Programa Familia Apoyo y Protección de la Red Básica de la FASC (PMPA). Correo electrónico: lourdes. sacarparo@terra.com. br. Neuza Maria de Fátima Guareschi, PhD. En Educación, University of Wisconsin - Madison, docente del departamento Psicología y del Programa de Posgrado en Psicología (PUCRS), Grupo de investigación Estudios Culturales y Teorías Contemporáneas. Correo electrónico: nmguares@pucrs.br. 
Unique System of the Social Attendance the family is prioritized in the law, we centered our discussion in the social programs of attention to the family, bringing discussions for the practices of the psychology.

Key-words: Public social politics, social attendance, family, psychology.

\section{Políticas públicas sociales}

Al hacer una retrospectiva, se observa que hasta finales del siglo XIX y principio del XX predominaban las ideas liberales de un Estado mínimo que solamente aseguraba el orden, la propiedad y el mercado como reguladores "naturales" de las relaciones sociales. La posición ocupada por el individuo en la sociedad y sus relaciones eran percibidas según su inserción en el mercado. "La cuestión social, decurrente del proceso productivo, se expresaba en la exclusión de las personas, tanto de la propia producción, como del usufructo de bienes y servicios necesarios a su propia producción" (Cunha \& Cunha, 2002, p. 11).

Después de la crisis económica de 1929 la cuestión social se intensificó y generó nuevas relaciones entre capital y trabajo, y entre estos dos y el Estado, lo cual trajo consigo que las élites económicas admitieran los límites del mercado como regulador natural y rescataran el papel del Estado como mediador civilizador, o sea, con poderes políticos de interferencia en las relaciones sociales. En este sentido, se puede entender la política social como estrategia de intervención y regulación del Estado en lo que se refiere a la cuestión social.

Demo (1996), coloca como telón de fondo la desigualdad social y afirma que la política social auténtica requiere implicar compromisos evidentes para reducirla. El autor es enfático al afirmar que la "política social necesita ser preventiva, en el sentido de ir a las raíces del problema evitando que se procese". Ya las "políticas curativas son inevitables frente a la pobreza vigente, pero no vencen el mal y pueden, en esa insistencia, incentivarlo" (p. 21).
Sin embargo, cuando el Estado toma para sí la responsabilidad de la formulación y ejecución de las políticas económica y social acaba convirtiéndose en "arena de disputas por acceso a la riqueza social", una vez que las políticas públicas involucran conflictos de intereses entre las clases sociales (Cunha $\&$ Cunha, 2002, p. 21). En ese proceso se destaca la participación de diversos movimientos que lucharon por garantizar derechos civiles, políticos y sociales. Muchas de las acciones del Estado fueron resultado de esas luchas. Así las cosas, "las políticas públicas han sido creadas como respuesta del Estado a las demandas que emergen de la sociedad y de su propio interior, siendo expresión del compromiso público de actuación en una determinada área a largo plazo" (p. 12). ¿Pero qué es "política pública"?

Según Pereira (1994), "es una línea de acción colectiva que concretiza derechos sociales declarados y garantizados en ley" (p. 59). Es mediante las políticas públicas que se distribuyen bienes y servicios sociales en respuesta a las demandas de la sociedad, caracterizándose como un derecho colectivo y no individual.

Veronese (1999) resalta que política pública no es sinónimo de asistencialismo ni, mucho menos, de paternalismo. "Es un conjunto de acciones, que forman una red compleja, dirigido hacia cuestiones precisas de relevancia social. Son acciones, en fin, que tienen como objetivo la promoción de la ciudadanía" (p. 193). Y el término "público" asociado a la política no es una referencia exclusiva al Estado, sino a la cosa pública, o sea, de todos, con el amparo de una misma ley y vinculado a una comunidad de intereses. De esa forma, Pereira (1994) resalta que, aunque las política públicas sean reguladas y frecuentemente proveídas por el 
Estado, ellas también engloban preferencias, escogencias y decisiones privadas que pueden ( $y$ deben) ser controladas por los ciudadanos. "La política pública expresa, así, la conversión de decisiones privadas en decisiones y acciones públicas, que afectan a todos" (p. 60).

Entre las diversas políticas públicas tales como la económica, la ambiental, la de ciencia y tecnología, "la política social es un tipo de política pública cuya expresión se da a través de un conjunto de principios, directrices, objetivos y normas, de carácter permanente e incluyente, que orientan la actuación del poder público en una determinada área" (Cunha \& Cunha, 2002, p. 12). Es importante destacar que la política social no puede ser confundida con ayuda, piedad o voluntariado.

En ese sentido entendemos que es un proceso de conciencia donde el suj eto se reconoce en su derecho, y la condición fundamental de enfrentamiento a la desigualdad es su propia actuación organizada. En palabras de Demo (1996): “la política social emancipadora es aquella que se funda en la ciudadanía organizada de los interesados. 0 sea, no trabaja con objetos manipulados sino con sujetos co-participantes y co-decisores" ( p. 26).

\section{La trayectoria de la asistencia social: el foco en la familia}

Vale la pena recordar que en las últimas décadas del siglo XX muchos países tuvieron que hacer fuerte ajuste económico una vez que la cuestión social se agravó por diversos factores como desempleo estructural, precarización de las relaciones de trabajo, alteraciones en la organización familiar y en el ciclo de vida (disminución de la tasa de mortalidad y aumento de la longevidad, por ej emplo) y el ahondamiento de las desigualdades sociales. Esto generó exclusión y, simultáneamente, inclusión marginal de gran fracción de la población (Cunha \& Cunha, 2002).

Las respuestas políticas de los diversos países a la cuestión social presentaron algunas medidas comunes, entre ellas el recorte de beneficios, ma- yor selectividad (no se aplica a todos) y la focalización de las políticas sociales, esto es, atender a los más pobres entre los pobres. Además, a través de la privatización de programas de bienestar social el Estado quedó exento de garantizar los mínimos sociales necesarios a la supervivencia humana.

En Brasil, la crisis que hubo como consecuencia del agotamiento del "milagro económico, al final de la década de 1970 e inicio de la década de 1980, propició una coyuntura socioeconómica favorable a los movimientos sociales de la sociedad civil. Según Cunha y Cunha (2002), el proceso de redemocratización de la sociedad brasileña llevó a la instalación de la Asamblea Nacional Constituyente y a la posibilidad de establecer otro orden social sobre nuevas bases, "lo que hizo que esos movimientos se articularan para intentar inscribir en la Carta Constitucional derechos sociales que pudieran ser traducidos como deberes del Estado a través de políticas públicas" (p. 13)". Es importante aclarar que este "milagro económico" es la denominación dada a la época de excepcional crecimiento económico ocurrido durante la dictadura militar. En ese período áureo del desarrollo brasileño en que, paradój icamente, hubo aumento de concentración de renta y de la pobreza. 0 sea, mientras más crecía el país, menor era el alcance de la clase trabajadora al crecimiento y la modernización.

Así, las décadas de 1980 y 1990 además de paradigmáticas fueron paradójicas en tanto que sirvieron de camino a una nueva configuración para el escenario político, económico y social brasileño. De un lado, se desarrolló un proceso singular de reformas en lo relativo a la ampliación del proceso democrático y a la organización política y jurídica, como la promulgación de la Constitución Federal en 1988, considerada por muchos como "guía de la tentativa de establecimiento de nuevas formas de relaciones sociales en el país" (Couto, 2004, p. 139). Por otro lado, se hizo efectivo un proceso de recesión y contradicciones en el campo económico, donde ocurrieron tentativas de disminuir la inflación galopante y se buscó retomar el crecimiento, teniendo como ejes los prin- 
cipios de macroeconomía expresada en la centralidad de la matriz económica en detrimento de la social.

La paradoja que se puede observar se localiza en la relación entre los avances sociales y las definiciones de las directrices macroeconómicas que conciben las políticas sociales como consecuencia del funcionamiento de la economía y, siendo así, acabaron por desfigurar los principios orientadores de las mismas. Fue exactamente en este contexto que se construyeron importantes y significativos avances "acarreando nuevas configuraciones y nuevos conceptos para el área de los derechos civiles, políticos y sociales, expresadas en la organización del sistema de seguridad social brasileño". Sto traj o para el área la asistencia social como una política social de naturaleza pública (Couto, 2004, p. 140).

La Constitución Federal de 1988 trajo un cambio para el concepto de asistencia social en el Brasil. Ésta pasa a constituir, junto con la salud, las cesantías y pensiones, la base de la seguridad social, notoriamente inspirada en la noción de Estado de Bienestar Social.

Este es un marco histórico que instituye el inicio de la transformación de la caridad, beneficio y ayuda en la noción de derecho y ciudadanía de la Asistencia Social, y señala su carácter de política pública de protección social articulada a otras políticas volcadas hacia la garantía de derechos y de condiciones dignas de vida. La asistencia social pasa a tener carácter universal aunque selectivo para quien la necesita.

En 1993 se da su reglamentación por la Ley Orgánica de la Asistencia Social (LOAS) en la cual la protección social se propone como un mecanismo contra las formas de exclusión social que son consecuencia de ciertas vicisitudes de la vida tales como la vejez, la enfermedad, la adversidad, las privaciones. Incluye en este concepto, también, tanto las formas selectivas de distribución y redistribución de bienes materiales (como comida y dinero), así como los bienes culturales (como los saberes) que permitirán la supervivencia y la inte- gración bajo varias formas en la vida social. De ese modo, la asistencia social se configura como posibilidad de reconocimiento público de la legitimidad de las demandas de sus usuarios y espacio de ampliación de su protagonismo.

En ese sentido, marca su especificidad en el campo de las políticas sociales y exige que las provisiones asistenciales sean prioritariamente pensadas en el ámbito de las garantías de ciudadanía bajo vigilancia del Estado; le compete al mismo la universalización de la cobertura y la garantía de derechos y acceso a servicios, programas y proyectos bajo su responsabilidad. La LOAS prevé también la creación de los Consejos de Asistencia Social, instancias deliberativas de composición paritaria que normalizan, acompañan y evalúan la política; la municipalización de la gestión de los recursos públicos financieros mediante los fondos de asistencia social y el reordenamiento institucional de la administración pública responsable por la coordinación, articulación y ej ecución de la política en cada esfera de gobierno (Costa, 2002).

Es importante resaltar que, en la esfera de la política de asistencia social, la LOAS considera como obj etivo la protección a la familia, y la determina como uno de los focos de atención de la política de asistencia social. Sin embargo, ¿Cómo entender las repercusiones de los procesos sociales y económicos en la familia ocurridos en la década de 1990? ¿Cuáles son los impactos de esas transformaciones en las condiciones de vida de los trabajadores, considerando la centralidad que la familia ocupa en el ámbito de la supervivencia material?

Podemos decir que los efectos de la crisis económica no hicieron posibles las reformas institucionales más amplias en los sistemas de protección social. Así, el derecho a la seguridad social garantizado en la Constitución no se hizo efectivo en lo que concierne a la asistencia social. Como consecuencia, vimos el ahondamiento de las desigualdades sociales y se constituyó, bajo nuevos parámetros, la exclusión social, o sea, el empobrecimiento de los trabajadores y sus familias 
(Alencar, 2004). Las medidas adoptadas para enfrentar esta situación fueron priorizar los programas focalizados (de carácter asistencialista), los fondos sociales de emergencia y los programas sociales compensatorios dirigidos hacia la atención de los grupos pobres y vulnerables. En este escenario colaboró para el agravamiento de la pobreza la tendencia histórica del sistema de protección social brasileño, o sea, la privatización en las áreas de la salud, educación y seguridad social.

A partir de estas consideraciones podemos decir que la introducción de la asistencia social como política del área de la seguridad social incorpora una innovación conceptual, pero también reitera las herencias históricas constitutivas de la cultura brasileña. Innova en la medida en que está respaldada tanto en el movimiento de la sociedad como en garantías legales pues integra las demás políticas de protección social. $Y$ en lo relativo al mantenimiento de los viejos conceptos históricos reitera la forma restrictiva de la misma, pues asocia esa área al asistencialismo y a las formas "de emergencia" de atender a la población; población que, en ese caso, es aquella vinculada a la pobreza absoluta (Pereira, 1996).

En ese sentido, Couto (2004) resalta que el campo de la asistencia social siempre fue un área nebulosa de la relación entre Estado y sociedad civil. Los conceptos de asistencialismo y clientelismo han sido señalados como constitutivos de una sociedad conservadora que por mucho tiempo (y aún hoy) ha considerado la pobreza como un atributo individual de aquellos que no se esforzaron para superarla. Para Alencar (2004) es una lógica cruel que culpabiliza a los individuos por su empleo y desempleo, y los responsabiliza por su formación profesional. Como no ha habido elevación significativa en las tasas de inversión, ni en el crecimiento económico, se tiene una situación en la cual el valor del trabajo (como fundamento de la ciudadanía social) "se subvierte frente a la creciente incapacidad del mercado de trabaj o formal de absorber el conjunto de la población potencialmente en condiciones de trabajar" (p. 74).
Para Castel (1998) en este contexto de creciente desempleo y precarización del trabajo lo más grave es la diversidad y la discontinuidad de las formas de empleo, fenómeno que produce vulnerabilidades sociales. El autor hace un paralelo entre el pauperismo del siglo XIX y las nuevas exigencias tecnológico-económicas de la evolución del capitalismo moderno. Es en eso que reside la gran transformación de las últimas décadas, por él denominada como metamorfosis del trabajo. 0 sea, confirma que "es sobre el trabajo - sea que se tenga, que falte, que sea precario o garantizado-que continúa desarrollándose hoy el destino de la gran mayoría de los actores sociales" (p. 156). Enfatizamos este cuadro social de creciente empobrecimiento de las familias brasileñas pues en el se conjugan falta de empleos, trabajo precario y deterioro de las condiciones y relaciones de trabajo. Así, el cotidiano de los trabajadores y sus familias está impregnado, muchas veces, por situaciones donde predomina la violencia en su modo de vida.

Sabemos que el trabajo tiene un significado de reconocimiento social para el sujeto. Hay quien afirma que el problema social se resuelve con trabajo. Si la idea de ciudadanía está enlazada a la obtención de trabajo, ¿cómo garantizarlo - para el conj unto de la población - frente al contexto antes descrito? Sumado a eso, el discurso de que la familia es la gran responsable por satisfacer la necesidad de sus miembros hace que la responsabilidad recaiga en ella. Es una lógica perversa pues además de no ocupar el espacio de pertenencia social, es responsabilizada por eso. ¿Esto es novedoso? No. Alencar (2004) señala que hay una tendencia histórica de resolver en la esfera privada cuestiones de orden público.

Castel (1998) afirma que al imponer las leyes de mercado al conjunto de la sociedad se da un proceso de destrucción de la forma específica de regulación social, mediada por el Estado, y esto hace que sobrevuele una amenaza que pone en primer plano la temática de la vulnerabilidad, como la exclusión. Aunque esa última expresión sea la más utilizada, el autor prefiere utilizar desafiliación pues ésta designa una trayectoria y el proceso que 
la está engendrando. El desafiliado parece ser el sujeto que no pertenece, aquel que no tiene lugar. Como oímos cotidianamente en las variadas interfaces de la red de atención a la infancia: la familia que no tiene condiciones, o suj eto que no tiene demanda, el niño que no sabemos hacia donde encaminar pues no hay lugar para él, como el niño portador de deficiencia y el niño suero-positivo o incluso el niño "que no es elegible para adopción".

En ese sentido, hacemos un paralelo con lo que Castel (1998) denomina como 'existencias dibuj adas en negativo', o sea, no son trabajadores, no tienen acceso a la protección de los derechos sociales garantizados por el Estado y no tienen calificaciones para el trabajo. Sabemos que el hambre no se refiere apenas a la comida concreta, sino también al hambre de ser filiado a un grupo que tenga visibilidad "en el positivo", de pertenecer, de ser reconocido, valorizado. Así, además de invertir en programas de renta mínima familiar y generación de renta, las estrategias de combate a la pobreza necesitan interferir en las relaciones de mercado una vez que son en éstas que se originan las condiciones de desigualdad social y acarrean la exclusión.

Estamos de acuerdo con Alencar (2004) cuando afirma que el Estado no puede devolverle a la familia la responsabilidad de las obligaciones que en realidad son responsabilidad del poder público. El autor señala que la familia debe ser priorizada en los programas sociales lo que, de cierta forma, se menciona en la Constitución Federal, en el Estatuto del Niño y del Adolescente, en la LOAS y, más recientemente, en el Sistema Único de Asistencia Social (SUAS). Veamos en qué consiste éste.

A partir de las deliberaciones de la IV Conferencia Nacional de la Asistencia Social se elabora el Plan Nacional de Asistencia Social y, aprobado por el Consejo Nacional de Asistencia Social (CNAS) en 2004, instituye el Sistema Único de la Asistencia Social que se propone como instrumento par la unificación de las acciones de la Asistencia Social, en el contexto nacional, materializando las direc- trices de la LOAS. En especial, ratifica el carácter de política pública de garantía de derechos y así se contrapone y destituye el histórico asistencialismo del "primer-damismo". Este nuevo modelo de gestión de la política de la Asistencia Social prioriza la familia como foco de atención y el territorio como base de la organización de acciones y servicios en dos niveles de atención: protección social básica y especial.

La Protección Social Básica tiene por obj etivo prevenir situaciones de riesgo a través del desarrollo de potencialidades y adquisiciones y el fortalecimiento de vínculos familiares y comunitarios. Se destina a la población que vive en situación de vulnerabilidad social consecuente de la pobreza, con precario acceso a los servicios públicos y/ o fragilización de vínculos afectivos. Prevé el desarrollo de servicios, programas y proyectos locales de acogida, convivencia y socialización de familias y de individuos, según la identificación de la situación de vulnerabilidad presentada.

La Protección Social Especial es la modalidad de atención destinada a familias e individuos que se encuentran en situación de riesgo personal y social debido a malos tratos físicos y/ o psíquicos, abuso sexual, cumplimiento de medidas socioeducativas, situación de trabajo infantil, entre otras. Las dificultades para ejercer funciones de protección fragilizan la identidad del grupo familiar y tornan más vulnerables sus vínculos simbólicos y afectivos.

\section{El lugar de la psicología en la asistencia social}

Todavía no hay una definición de actividades específicas del psicólogo, pero las primeras situaciones encontradas en el trabajo cotidiano y sus consecuencias emocionales, como situaciones de abuso sexual y violencia doméstica, trajeron una disyuntiva: ¿serán causa o consecuencia? Frente a evaluaciones del tipo "madre negligente", en el caso de niños que son llevados a la institución sucios, se contrapone el contexto de no tener ducha o agua caliente, ante lo cual no bañarlo en un día 
frío de invierno puede ser una forma de protección.

La metodología de trabajo propuesta por los profesionales de la psicología, en principio, no llega a los usuarios de los servicios y, por lo tanto, es cuestionada. Frente al discurso según el cual el psicólogo no hace visita domiciliaria, asociado a un "pretendido método clínico" y la resistencia al trabajo con grupos, la propia clínica es cuestionada pues aquel no es el lugar del consultorio particular. Es la propia "mirada" de la psicología lo que se cuestiona, cuando se queda en la apariencia, en el contraste con los valores y códigos personales, y acaba, entonces, por reafirmar la exclusión ("no puedo atender, no hay demanda") en vez de provocar lecturas posibles del proceso de exclusión con propuestas de acciones de inclusión (como "buscar" quién está excluido y/ o se excluye). En ese sentido, ¿será que el psicólogo produce demanda de atención al tratarse de la especificidad de la clientela de la asistencia social? ¿De qué especificidad se está hablando? ¿Cómo se constituye demanda?

Para algunos profesionales, el psicoanálisis les serviría de apoyo para pensar el sujeto a partir de una escucha del discurso y hacer el reconocimiento de lugar social a partir de la idea de la clínica ampliada. Si bien esta toda esta cuestión parece nueva, fue ya prevista por Freud en el inicio del siglo XX, cuando señaló el deber del Estado no sólo en la asistencia a la salud mental sino en su asociación con la asistencia social.

Se nos presentará entonces la tarea de adaptar nuestra técnica a las nuevas condiciones. [...] Tendremos que buscar la expresión más sencilla y comprensible de nuestras teorías. [...] La vida pesada que les espera (pobres) no les ofrece atractivo alguno y la enfermedad les confiere un derecho más a la asistencia social. Es probable que solamente consigamos obtener algún resultado cuando podamos unir al socorro psíquico un auxilio material. [...] Pero, cualesquiera que sean la estructura y la composición de esta psicoterapia para el pueblo, sus elementos más importantes y eficaces continuarán siendo sacados del psicoanálisis propiamente dicho, riguroso y exento de toda idea tendenciosa (Freud, 1918, p. 312).

Esta discusión sobre el lugar del psicólogo, sea en la salud o en la asistencia, y las primeras interfaces con las políticas públicas, es antigua y al mismo tiempo actual. La diferencia es que las discusiones están dej ando de tener carácter informal para ocupar territorios legitimados como las publicaciones, los congresos y los simposios, así como grupos de estudios para discutir cuestiones relativas a la psicología y a las políticas públicas.

EI SUAS propone como alternativa hacer efectiva la implantación del Programa de Atención Integral a la Familia (PAIF), que es un servicio continuado de protección social básica desarrollado en los Centros Regionales de Asistencia Social. Esos centros son espacios físicos localizados estratégicamente en áreas de pobreza para prestar atención socioasistencial, son los encargados de articular los servicios disponibles en cada localidad y potencializan la red de protección social básica. El PAIF se destina a promover el acompañamiento de familias en una determinada región (territorialización), potencializa la familia como unidad de referencia fortaleciendo vínculos internos y externos de solidaridad, contribuye al proceso de autonomía y emancipación social de las familias fomentando su protagonismo, desarrolla acciones que involucren diversos sectores con el objetivo de romper el ciclo de reproducción de la pobreza entre generaciones y actúa de forma preventiva evitando que esas familias tengan sus derechos violados recayendo en situaciones de riesgo. Esas familias, como consecuencia de la pobreza, están vulnerables, privadas de renta y de acceso a los servicios públicos, con vínculos afectivos frágiles, son discriminadas por cuestiones de género, etnia, deficiencia, edad, entre otras.

En el municipio de Porto Alegre el PAIF obtuvo la aprobación de su proyecto de ejecución en 2004 focalizado en la potencialización de la red. Se cons- 
tató que algunas familias que venían siendo atendidas a través de programas de orientación y apoyo sociofamiliar continuaban en situación de vulnerabilidad, principalmente en lo que se refiere a las niños en los semáforos. Sin embargo, el trabajo que niños y adolescentes realizaban durante las vacaciones era monitoreado a través de denuncias, puesto que el mismo se entendía como comportamiento de riesgo, principalmente cuando estos niños y adolescentes estaban ubicados en el centro de la ciudad. Por otro lado, se pudo constatar que el sufrimiento psíquico de algunas familias les impedía realizar movimientos que les Ilevaran a la emancipación social.

Cabe aquí cuestionarnos sobre cuál es la diferencia entre encaminar una familia para la atención en salud mental, como psicoterapia o terapia familiar, y la medida de protección legal de orientación y apoyo sociofamiliar. La cuestión parece estar en la identificación de situación de violación de derechos del niño o adolescente, como abuso sexual, maltratos y negligencia, al gunas veces asociada a dependencia de substancias psicoactivas, o trabajo infantil, o situación de calle, para citar algunos ej emplos. Nos interrogamos especialmente cuando la familia no busca ayuda por sí misma. Cuando es necesario que un "tercero" intervenga, sea la escuela, el centro de salud, la comunidad directamente 0 a través de los operadores de derecho como el Ministerio Público (Fiscalía) y J uzgado de la Infancia y J uventud. La demanda, en ese caso, se coloca afuera, a partir de otro, y el trabajo se basará en la constitución de la demanda de tratamiento. Muchas veces la orientación dada no es cumplida por la familia, lo cual genera una determinación legal, e inclusive una medida de amparo del niño o adolescente. Así, ¿cómo trabajar el retorno a la familia una vez está implícito que ésta haga movimientos de adhesión y permanencia en tratamiento?

También nos preguntamos hasta qué punto las cuestiones materiales dificultan la escucha de la historia y de los significados del sujeto. Si las familias vienen a la Atención a la Comunidad y hablan de las carencias de comida, de remedio, de casa, de ropa, de material escolar, de trabajo y de ayuda, cuando se pregunta más allá muchas veces hablan de des-reconocimiento social, de falta de lugar, de falta de origen. Si para el Psicoanálisis tradicionalmente se dice que no está en cuestión la realidad objetiva sino la subjetiva, pensamos que en la cuestión de la miseria, extrema pobreza y exclusión social es necesario tener en cuenta el contexto.

En lo que se respecta a la atención a las familias de las clases populares, Carvalho (2002) afirma que hay una tendencia de los servicios a tomar los sujetos/ familias como portadores de una única historia, de un patrón y son catalogados como dependientes y excluidos. Concordamos con la autora al señalar la importancia de la escucha y del acogimiento pues solo así se aclara la demanda. Y, para nosotros, acoger implica escuchar, aceptar la verdad subjetiva para el sujeto sin crítica o juicio. Se torna imprescindible entonces revisar los parámetros que muchas veces utilizamos para hacer evaluaciones psicosociales. ¿Estamos considerando la cultura y los valores de la comunidad?

Para eso se hace necesario construir una relación dialógica en la que se procure identificar los potenciales emancipatorios del grupo familiar y de la comunidad. Quien sabe si al estar el profesional abierto a escuchar otras formas de subj etivación, otros modos de protección de los hijos, estaremos construyendo caminos que posibiliten potencializar los sujetos. No tener la expectativa de cambiar las familias sino facilitar los cambios obj etivando la emancipación puede ser el inicio de la horizontalidad de las relaciones.

Sin embargo, será que la posibilidad de escucha no se reduce cuando recae sobre el psicólogo también el lugar de controlador, el profesional a quien la familia rinde cuentas. Este es el interrogante de las psicólogas Rispoli, Viñas y Susin (2004). Se percibe entonces que las vulnerabilidades no se superan, apenas, satisfaciendo las necesidades materiales. Sustentar la escucha de la subjetividad del usuario frente a la violación de derechos del niño y del adolescente no es tarea fácil. Por 
ej emplo, cuando un niño está fuera de la escuela y eso no es problema para su madre. Nuevamente nos remite a una cuestión de lugar.

El dilema es cómo operar en dos sentidos: del lado del derecho, al considerar la ley jurídica, que señala hacia la normatización de las conductas, 0 del lado de la escucha al considerar el punto de vista de la verdad del sujeto․ Paradójicamente, la psicología mirará (o no) hacia otros factores como relativizar los actos del sujeto y escuchar desde donde él se sitúa frente a esta violación, por ejemplo, tomar en consideración cómo se organizan los lazos familiares, qué principios y valores rigen este grupo familiar. Como dice Gonçalves (2003, p. 143), "en la red social, de nada vale pretender que el código jurídico, per se, enjaule la conducta: la ley solo podrá producir cambios reales cuando sea capaz de atender a la cultura local". En ese sentido "muchas acciones han sido adoptadas sin llevar en cuenta el sustrato cultural sobre el cual se implementan, en flagrante desconsideración de las representaciones locales sobre la infancia, familia, autoridad paterna y educación".

El desafío está planteado: ¿cómo trabajar con los programas de orientación y apoyo sociofamiliar de forma que promuevan la autonomía y los derechos de la familia sin que esta acción se transforme en un vínculo más de control? ¿Estos programas serían los nuevos aparatos de la "Policía de las Familias" ??. Muchas acciones se configuran en dispositivos de control sobre las familias y los sujetos, exactamente como se vio con la figura del criminal (Foucault, 1984) y con los dispositivos higiénicos que fundaron la familia nuclear moderna (Donzelot, 2001).
Puesto que a partir del SUAS se prevé en los Centros de Referencia de la Asistencia Social (CRAS) y en los Centros de Referencia Especializados de Asistencia Social (CREAS) la presencia del profesional de psicología en la composición del equipo mínimo, nos preguntamos ¿cuáles son las prácticas de la psicología en estos Centros?

El Consej o Federal de Psicología ha promovido una investigación on-line a través del Centro de Referencia Técnica en Psicología y Políticas Públicas con el objetivo de construir colectivamente las referencias técnicas para la actuación profesional en las políticas públicas brasileñas. En aras de contribuir en ese debate cabe cuestionarse, a partir de las reflexiones propuestas por Cruz (2006), qué interpretación y operacionalización de acciones se efectuarán en la articulación de la red básica y especializada de servicios socio-asistenciales cuando una acción de amparo temporal para un niño pequeño es requerida en los llamados casos de "negligencia", donde la seguridad del niño requiere un amparo en tiempo integral y al mismo tiempo la inversión en el trabajo con la presencia de los padres. ¿Como podrían constituirse centros de convivencia donde el niño pudiese permanecer día y noche y que facilitasen las visitas diarias de los padres o familiares? ¿Las "familias substitutas" serían una acción de protección básica o especializada, o serían una acción especializada en la atención básica? La preocupación que esta dicotomía entre básica y especializada nos propone tiene raíces en las prácticas históricas pues aún las mejor intencionadas muchas veces resultarán en el indeseado refuerzo de la institucionalización.

Estas cuestiones fueron propuestas por las psicólogas J anete Constantinou, Marisa Warpechowski y Luciane Susin en el "Debate sobre la Psicología en la Asistencia Social" promovido por la Comisión de Políticas Públicas del Consejo Regional de Psicología de Rio Grande do Sul el día 16/05/ 05.

2 Donzelot (2001) en el libro "La Policía de las Familias" discute cómo en la Francia de los siglos XVIII y XIX lo social se centraba alrededor de la familia, de sus ej ercicios y deberes. Bajo el pretexto de promover la asistencia a los segmentos pobres, y utilizando la producción de un sentimiento de responsabilidad social, se elige el control y la disciplinarización para hacer valer el orden económico-político-social vigente que favorecía los intereses de la burguesía amenazados por la creciente miseria. De esa forma la ascensión de lo social promoverá la producción de modelos de familia, educación y trabajo. 
Si la política de asistencia social se ve como un proceso de travesía para garantizar el acceso a las demás políticas públicas, ésta ha tenido que "nadar contra la corriente". La pobreza muchas veces aún es percibida como un atributo individual de aquellos que no se esforzaron en superarla. Son pocas las personas que se acercan a la asistencia social para garantizar sus derechos y muchas veces son vistas como "limosneros". Para la psicología comprometida con el reconocimiento de las singularidades y la promoción de la autonomía, de la superación del trabajo infantil de los niños en los semáforos y, en fin, con intervenciones asertivas que garanticen los derechos establecidos por ley, supone un cambio de miradas y con certeza un avance en la integración de la red que lleve su texto a las últimas consecuencias en el sentido del cambio por la vía de la integración. De lo contrario, se corre el riesgo de ser más un sistema que sólo se ve bonito en el papel.

\section{Referencias}

Alencar, M. M. T. (2004). Transformações econômicas e sociais no Brasil dos anos 1990 e seu impacto no âmbito da família. Em: M. A. Sales, M.C. Matos \& M.C. Leal (Orgs.), Política social, família e juventude: uma questão de direitos. São Paulo: Cortez.

Constituição da República Federativa do Brasil. (1988). Brasília/ DF.

Carvalho, M. C. B. (2002). A priorização da família na agenda da política social. Em: S.M. Kaloustian (Orgs.) Família brasileira, a base de tudo. 5.ed. São Paulo: Cortez; Brasília, UNICEF.

Castel, R. (1998). As metamorfoses da questão social: uma crônica do salário. Petrópolis: Vozes.

Costa, B.L.(2002). As mudanças na agenda das políticas sociais no Brasil e os desafios da inovação: o caso das políticas de assistência social à infância e a adolescência. Em: A.
Carvalho; et al. (Orgs), Políticas Públicas. Belo Horizonte: UFMG; PROEX.

Couto, B. R. (2004). O direito social e a assistência social na sociedade brasileira: uma equação possível? São Paulo: Cortez.

Cruz, L R. da. (2006). (Des)articulando as políticas públicas no campo da infância: implicações da abrigagem. Santa Cruz do Sul: EDUNISC.

Cunha, E.P. \& Cunha, E.S. (2002). Políticas públicas sociais. Em: A. Carvalho; et al. (Orgs), Políticas Públicas. Belo Horizonte: UFMG; PROEX.

Demo, P. (1996). Política social, educação e cidadania. Campinas: Papirus.

Diário Oficial da República Federativa do Brasil. (1993). Lei Orgânica da Assistência Social, Lei 8.742, de 7 de dez. 1993. Brasília/ DF.

Diário Oficial da República Federativa do Brasil. (1990). Estatuto da Criança e do Adolescente, Lei 8.069, de 13 jul. 1990. Brasília/ DF.Brasil.

Foucault, M. (1984). Vigiar e punir: hsitória da violência nas prisões (3a ed.) Petrópolis: Vozes.

Donzelot, J. (2001). A polícia das famílias. (3ạ ed.) Rio de J aneiro: Graal.

Freud, S. (1918). Caminhos da terapêutica psicanalítica. Em: Obras Completas de Sigmund Freud, vol. X. Rio de J aneiro: Delta.

Gonçalves, H.S. (2003). Infância e violência no Brasil. Rio de J aneiro: NAU/ FAPERJ .

Ministério do Desenvolvimento Social e Combate à Fome - Secretaria Nacional de Assistência Social Brasil (2004). Política Nacional de Assistência Social. Brasília/ DF. 
Pereira, P. A. (1994). Concepções e propostas de políticas sociais em curso: tendências, perspectivas e conseqüências. Brasília: NEPPOS/ CEAM/ UnB.

Pereira, P. A. (1996). A assistência social na perspectiva dos direitos: crítica aos padrões dominantes de proteção social aos pobres no Brasil. Brasília: Thesaurus.
Rispoli, A., Vinãs, A.C. \& Susin, L.M. (2004). A Psicologia na assistência social: desafios e reflexões. Em: I Seminário Regional de Práticas Sociais: Rede de Proteção à Criança e ao Adolescente em Situação de Risco Social. Fundação de Assistência Social e Cidadania (Org.). Porto Alegre: Evangraf.

Veronese, J. R. P. (1999). Os direitos da criança e do adolescente. São Paulo: LTR. 